\title{
A Survey on Public Auditing for Integrity Checking and Regenerating Faulty Data Block in Cloud Data Storage
}

\author{
Shamala M G ${ }^{1}$, Rekha K B ${ }^{2}$ \\ ${ }^{1}$ M. Tech, Department Of Computer Science, Rajiv Gandhi Institute of Technology \& Engineering, Bangalore, India \\ ${ }^{2}$ Assistant professor, Department Of Computer Science, Rajiv Gandhi Institute of Technology \& Engineering, Bangalore, India
}

\begin{abstract}
To ensure outsourced information in distributed storage against debasements, adding adaptation to non-critical failure to distributed storage together with information honesty checking and disappointment reparation gets to be basic. As of late, recovering codes have picked up fame because of their lower repair transmission capacity while giving adaptation to non-critical failure. Existing remote checking techniques for recovering coded information just give private reviewing, requiring information proprietors to dependably stay online and handle evaluating, and in addition repairing, which is here and there unreasonable. In this paper, we propose an open reviewing plan for the recovering code-based distributed storage. To tackle the recovery issue of fizzled authenticators without information proprietors, we present an intermediary, which is advantaged to recover the authenticators, into the conventional open examining framework model. Besides, we plan a novel open evident authenticator, which is produced by a few keys and can be recovered utilizing incomplete keys. Hence, our plan can totally discharge information proprietors from online weight. Furthermore, we randomize the encode coefficients with a pseudo irregular capacity to protect information security. Broad security examination demonstrates that our plan is provable secure under arbitrary prophet model and test assessment shows that our plan is exceptionally effective and can be practically coordinated into their producing code-based distributed storage.
\end{abstract}

Keywords: Cloud storage, regenerating codes, public audit, privacy preserving, authenticator regeneration, proxy, privileged, provable secure

\section{Introduction}

Distributed storage is presently picking up ubiquity since it offers an adaptable on-interest information outsourcing administration with engaging advantages alleviation of the weight for capacity administration, all inclusive information access with area freedom, and evasion of capital use on equipment, programming, and individual systems for upkeeps, and so forth., In any case, this new worldview of information facilitating benefit likewise brings new security dangers toward client's information, subsequently making people or enterprisers still feel reluctant. It is noticed that information proprietors lose extreme control over the destiny of their outsourced information; along these lines, the accuracy, accessibility and respectability of the information are being put at danger.

From one perspective, the cloud administration is typically confronted with an expansive scope of interior/outer foes, who might noxiously erase or degenerate clients' information; then again, the cloud administration suppliers may act unscrupulously, endeavoring to shroud information misfortune or defilement and guaranteeing that the documents are still effectively put away in the cloud for notoriety or fiscal reasons. In this way it bodes well for clients to execute proficient convention to perform periodical checks of their outsourced information to ensure that the cloud in reality keeps up their information accurately.

Numerous instruments managing the honesty of outsourced information without a neighborhood duplicate have been proposed under various framework and security models up to now. The most critical work among these studies are the PDP (Provable Data possession)is a provable information ownership model and POR (Proof of retriveability) confirmation of recover capacity model, which were initially proposed for the single-server situation by A tenieseet al. also, Juels and Kaliski [3], separately. Considering that documents are normally striped and needlessly put away crosswise over multi-servers or multi-mists, investigate trustworthiness check plans suitable for such multi-servers or multi-mists setting with various excess plans, for example, replication, eradication codes, and, all the more as of late, recovering codes.

In this paper, we concentrate on the respectability check issue in recovering code-based distributed storage, particularly with the utilitarian repair methodology the flawed servers. Considering the substantial size of the outsourced information and the client's compelled asset capacity, the errands of inspecting and reparation in the cloud can be impressive and expensive for the clients. The overhead of utilizing distributed storage should be minimized however much as could reasonably be expected such that a client does not need to perform too numerous operations to their outsourced data (in extra to recovering it). Specifically, clients may not want to experience the unpredictability in confirming and reparation.

The examining plans in infer the issue that users need to dependably stay on the web, which may obstruct its adoption by and by, particularly for long haul documented storage. To completely guarantee the information trustworthiness and recovery the users' computation assets and in addition online weight, we propose an open inspecting plan for the 


\section{International Journal of Science and Research (IJSR) \\ ISSN (Online): 2319-7064}

Index Copernicus Value (2013): 6.14 | Impact Factor (2015): 6.391

recovering code-based distributed storage, in which the respectability checking and regeneration(of fizzled information pieces and authenticators) are executed by an outsider inspector and a semi-trusted intermediary independently for the benefit of the information proprietor

Rather than specifically adjusting the current open reviewing plan to the multi-server setting, we outline a novel authenticator, which is more appropriate for recovering codes. In addition, we "scramble" the coefficients to ensure information security against the inspector, which is more lightweight than applying the confirmation blind technique in and information blind strategy in.

Several challenges and dangers suddenly emerge in our new system model with an intermediary and security examination shows that our plan functions admirably with these problems. We outline a novel homomorphic authenticator based on BLS signature, which can be produced by a couple of mystery keys and confirmed freely. Using the straight subspace of the recovering codes, the authenticators can be registered effectively. In addition, it can be adjusted for information proprietors furnished with low end calculation devices (e.g. Tablet PC and so forth.) in which they just need to sign the local squares.

- To the best of our insight, our plan is the first to permit security protecting open reviewing for regenerating codebased distributed storage. The coefficients are masked by a PRF (Pseudorandom Function) amid the Setup phase to maintain a strategic distance from spillage of the first information. This method is lightweight and does not acquaint any computational overhead with the cloud servers or TPA.

- Our plan totally discharges information proprietors from online burden for the recovery of squares and authenticators at broken servers and it gives the benefit to a proxy for the reparation.

- Optimization measures are taken to enhance the flexibility and productivity of our examining plan; along these lines, the storage overhead of servers, the computational overhead of the data proprietor and correspondence overhead amid the audit phase can be successfully lessened.

- Our plan is provable secure under irregular oracle model. Moreover, we make a correlation with the condition of the art and tentatively assess the execution of our plan.

\section{Literature Survey}

The problem of remote data checking for integrity was introduced in [1] and [2], provable data possession (PDP) and proof of retrievability (POR), respectively. In [2] proposed a formal definition of the PDP model for ensuring possession of files on untrusted storage, introduced the concept of RSA-based homomorphic tags and suggested randomly sampling a few blocks of the file. To release the data owner from online burden for verification, [2] considered the public auditability in the PDP model for the first time. However, their variant protocol exposes the linear combination of samples and thus gives no data privacy guarantee. Then in, [4] developed a random blind technique to address this problem in their BLS signature based public auditing scheme. Similarly, [5] introduced another privacy- preserving method, which is more efficient since it avoids involving a computationally intensive pairing operation for the sake of data blinding. In [6] presented a public PDP scheme, where the data privacy is provided through combining the cryptography method with the bilinearity property of bilinear pairing. [7] Utilized random mask to blind data blocks in error-correcting coded data for privacypreserving auditing with TPA. In [8] proposed a formal framework for interactive provable data possession (IPDP) and a zero-knowledge IPDP solution for private clouds. Their ZK-IPD protocol supports fully data dynamics, public verifiability and is also privacy-preserving against the verifiers. To introduce fault tolerance in practical cloud storage, outsourced data files are commonly striped and redundantly stored across multi-servers or even multiclouds. It is desired to design efficient auditing protocols for such settings, specifically in [9]. In [10] make great effort to design auditing schemes for regenerating-code-based cloud storage, which is similar to our contribution except that ours release the data owner from online burden for verification and regeneration. Furthermore, in [11] proposed an efficient construction of cooperative provable data possession (CPDP) which can be used in multi-clouds, and [12] extend their primitive auditing protocol to support batch auditing for both multiple owners and multiple clouds.

\section{Objective of the Project \& Description}

- Efficient checking of data integrity

- Efficient support for repairing failed nodes

- Protection against information leakage, when checking is performed by a third party.

\section{Design Phase}

System Design

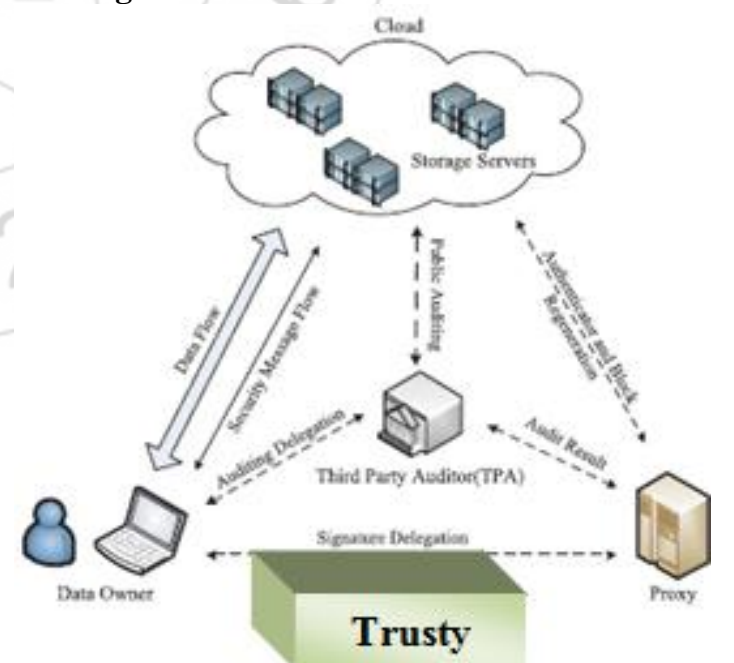

Figure 1: System Model

We consider the examining framework model for Regenerating-Code-based distributed storage as Fig.1, which includes five elements: the information proprietor, who claims a lot of information documents to be put away in the cloud; the cloud, which are overseen by the cloud administration supplier, give stockpiling benefit and have noteworthy computational assets; the outsider evaluator Third Party Auditor (TPA), who has ability and capacities to 


\section{International Journal of Science and Research (IJSR) \\ ISSN (Online): 2319-7064}

Index Copernicus Value (2013): 6.14 | Impact Factor (2015): 6.391

lead open reviews on the coded information in the cloud, the TPA is trusted and its review result is unprejudiced for both information proprietors and cloud servers; the intermediary specialists, who is semi-trusted and follows up in the interest of the information proprietor to recover authenticators and information hinders on the fizzled servers amid the repair technique. Trusty Notice that the information proprietor is confined in computational and capacity assets contrasted with different elements and may get to be logged off even after the information transfer strategy. The intermediary, who might dependably be on the web, should be considerably more capable than the information proprietor however not exactly the cloud servers as far as calculation and memory limit. To spare assets and additionally the online weight conceivably brought by the occasional examining and incidental repairing, the information proprietors resort to the TPA for respectability check and delegate the reparation to the intermediary. Contrasted and the conventional open examining framework model, the trusty agent is maintained between data owner and a proxy agent. The function of this module is to give authentication for data owner and a proxy agent, in order to establish the connection and data transfer between them.

Our framework model includes extra intermediary operators. So as to uncover the discernment of our outline and make to be more clear and solid, we think about, for example, a reference situation: An organization utilizes a business recovering code-based open cloud and gives long haul recorded capacity administration for its staffs, the staffs are outfitted with low end calculation gadgets (e.g., Laptop PC, Tablet PC, and so on.) and will be much of the time disconnected from the net. For open information inspecting, the organization depends on a trusted outsider association to check the information uprightness; Similarly, to discharge the staffs from overwhelming online weight for information and authenticator recovery, the organization supply an intense workstation (or bunch) as the intermediary and give intermediary reparation administration to the staffs' information.

\section{Definitions of Our Auditing Scheme}

The auditing scheme consists of three procedures:

Setup, Audit and Repair phase. Each procedure contains certain polynomial-time algorithms as follows:

Setup: The data owner maintains this procedure to initialize the auditing scheme.

- Key Gen $\left(\mathbf{1}^{\mathbf{k}}\right) \rightarrow$ (pk, sk): This polynomial-time algorithm is run by the data owner to initialize its public and secret parameters by king a security parameter $\kappa$ as input.

- Delegation (sk) $\rightarrow(\mathbf{x}):$ This algorithm represents the interaction between the data owner and proxy. The data owner delivers partial secret key $x$ to the proxy through a secure approach.

- Sig And Block Gen(sk, F) $\rightarrow(\Phi, \psi, t)$ : This polynomial time algorithm is run by the data owner and takes the secret parameter $s k$ and the original file $F$ as input, and then outputs a coded block set, an authenticator set and a file tag $t$.
Audit: The cloud servers and TPA interact with one another to take a random sample on the blocks and check the data intactness in this procedure.

- Challenge (F info) $\rightarrow(\mathbf{C})$ : This algorithm is performed by the TPA with the information of the file $F$ info as input and a challenge $C$ as output.

- Proof $\operatorname{Gen}(\mathbf{C}, \Phi, \psi) \rightarrow(\mathbf{P})$ : This algorithm is run by each cloud server with input challenge $C$, coded block set and authenticator set, then it outputs a proof $P$.

- Verify $(P, p k, C) \rightarrow(0,1)$ : This algorithm is run by TPA immediately after a proof is received. Taking the proof $P$, public parameter $p k$ and the corresponding challenge $C$ as input, it outputs 1 if the verification passed and 0 otherwise.

Repair: In the absence of the data owner, the proxy interacts with the cloud servers during this procedure to repair the wrong server detected by the auditing process.

- Claim for $\operatorname{Rep}(\mathbf{F}$ info) $\rightarrow(\mathbf{C r})$ : This algorithm is similar with the Challenge () algorithm in the Audit phase, but outputs a claim for repair $C r$.

- Gen for $\operatorname{Rep}(\mathbf{C r}, \Phi, \psi) \rightarrow(\mathbf{B A}):$ The cloud servers run this algorithm upon receiving the $\mathrm{Cr}$ and finally output the block and authenticators set $B A$ with another two inputs.

- Block and Sig $\operatorname{ReGen}(\mathbf{C r}, \mathbf{B A}) \rightarrow\left(\Phi^{\prime}, \psi^{\prime}, \perp\right):$ The proxy implements this algorithm with the claim $\mathrm{Cr}$ and responses $B A$ from each server as input, and outputs a new coded block

- Set $\psi$ and authenticator set $\Phi$ if successful, outputting $\perp$ if otherwise.

\section{Conclusion}

In this paper, we propose a public auditing scheme for the regenerating-code-based cloud storage system, where the data owners are privileged to delegate TPA for their data validity checking. To protect the original data privacy against the TPA, we randomize the coefficients in the beginning rather than applying the blind technique during the auditing process. Considering that the data owner cannot always stay online in practice, in order to keep the storage available and verifiable after a malicious corruption, we introduce a semi-trusted proxy into the system model and provide a privilege for the proxy to handle the reparation of the coded blocks and authenticators. The trusty agent is maintained between data owner and a proxy agent. The function of this module is to give authentication for data owner and a proxy agent, in order to establish the connection and data transfer between them. Authenticator can be efficiently generated by the data owner simultaneously with the encoding procedure.

\section{References}

[1] G. Ateniese et al., "Provable data possession at untrusted stores", in Proc. 14th ACM Conf. Comput. Commun. Secur. (CCS), New York, NY USA, 2007, pp. 598-609.

[2] Ari Juels and Burton S. Kaliski Jr., "PORs: Proofs of Retrievability for Large Files", in Proc. 14th ACM 


\section{International Journal of Science and Research (IJSR) \\ ISSN (Online): 2319-7064}

Index Copernicus Value (2013): 6.14 | Impact Factor (2015): 6.391

Conf. Comput. Commun. Secur. (CCS), New York, NY USA, 2007, pp. 584-597.

[3] Mehul A.Shah, Mary Baker, jeffrey C. Mogul, "Auditing to keep online storage services honest", in Proc. Of hotOS'07, 2007, pp.1-6.

[4] C. Wang, Q. Wang, K. Ren, and W. Lou, "Privacypreserving public auditing for data storage security in cloud computing," in Proc. IEEE INFOCOM, Mar. 2010, pp. 1-9.

[5] S. G. Worku, C. Xu, J. Zhao, and X.He, "Secure and efficient privacypreserving public auditing scheme for cloud storage," Comput. Elect. Eng., vol. 40, no. 5, pp. 1703-1713, 2013.

[6] K. Yang and X. Jia, "An efficient and secure dynamic auditing protocol for data storage in cloud computing," IEEE Trans. Parallel Distrib. Syst., vol. 24, no. 9, pp. 1717-1726, Sep. 2013.

[7] C. Wang, Q. Wang, K. Ren, N. Cao, and W. Lou, "Toward secure and dependable storage services in cloud computing," IEEE Trans. Service Comput., vol. 5, no. 2, pp. 220-232, Apr./Jun. 2012.

[8] Y. Zhu, H. Hu, G.-J. Ahn, and M. Yu, "Cooperative provable data possession for integrity verification in multicloud storage," IEEE Trans. Parallel Distrib. Syst., vol. 23, no. 12, pp. 2231-2244, Dec. 2012.

[9] R. Curtmola, O. Khan, R. Burns, and G. Ateniese, "MRPDP: Multiple-replica provable data possession," in Proc. 28th Int. Conf. Distrib. Comput. Syst. (ICDCS), Jun. 2008, pp. 411-420.

[10] B. Chen, R. Curtmola, G. Ateniese, and R. Burns, "Remote data checking for network coding-based distributed storage systems," in Proc. ACM Workshop Cloud Comput. Secur. Workshop, 2010, pp. 31-42.

[11] A. G. Dimakis, K. Ramchandran, Y. Wu, and C. Suh, "A survey on network codes for distributed storage," Proc. IEEE, vol. 99, no. 3, pp. 476-489, Mar. 2011.

[12] H. C. H. Chen and P. P. C. Lee, "Enabling data integrity protection in regenerating-coding-based cloud storage: Theory and implementation," IEEE Trans. Parallel Distrib. Syst., vol. 25, no. 2, pp. 407-416, Feb. 2014.

[13] J. He, Y. Zhang, G. Huang, Y. Shi, and J. Cao, "Distributed data possession checking for securing multiple replicas in geographicallydispersed clouds," $J$. Comput. Syst. Sci., vol. 78, no. 5, pp. 1345-1358, 2012.

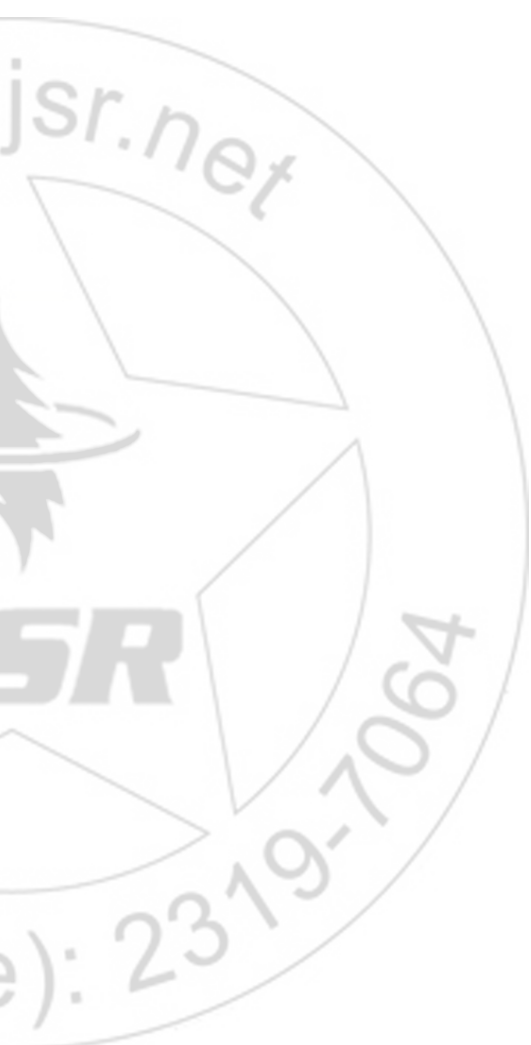

